\title{
Genotyping in 46 patients with tentative diagnosis of Treacher Collins syndrome revealed unexpected phenotypic variation
}

Özge Altug Teber ${ }^{1}$, Gabriele Gillessen-Kaesbach ${ }^{1}$, Sven Fischer ${ }^{1}$, Stefan Böhringer ${ }^{1}$, Beate Albrecht ${ }^{1}$, Angelika Albert ${ }^{2}$, Mine Arslan-Kirchner ${ }^{3}$, Eric Haan ${ }^{4}$, Monika Hagedorn-Greiwe ${ }^{5}$, Christof Hammans ${ }^{6}$, Wolfram Henn ${ }^{7}$, Georg Klaus Hinkel ${ }^{8}$, Rainer König ${ }^{9}$, Erdmute Kunstmann ${ }^{6}$, Jürgen Kunze ${ }^{10}$, Luitgard M Neumann ${ }^{10}$, Eva-Christina Prott ${ }^{1}$, Anita Rauch ${ }^{11}$, Hans-Dieter Rott ${ }^{11}{ }^{1}$, Heide Seidel $^{2}$, Stephanie Spranger ${ }^{12}$, Martin Sprengel $^{13}$, Barbara Zoll ${ }^{14}$, Dietmar R Lohmann*,1 and Dagmar Wieczorek ${ }^{1}$

${ }^{1}$ Institut für Humangenetik, Universitätsklinikum, Essen, Germany; ${ }^{2}$ Abteilung für pädiatrische Genetik der Kinderpoliklinik, Ludwig-Maximilian-Universität, München, Germany; ${ }^{3}$ Institut für Humangenetik, Medizinische Hochschule, Hannover, Germany; ${ }^{4}$ South Australian Clinical Genetics Service, Women's \& Children's Hospital, Adelaide, Australia; ${ }^{5}$ Institut für Humangenetik, Universitätsklinikum, Lübeck, Germany; ${ }^{6}$ Humangenetik, RuhrUniversität, Bochum, Germany; ${ }^{7}$ Institut für Humangenetik, Universität des Saarlandes, Homburg/Saar, Germany; ${ }^{8}$ Institut für Klinische Genetik, Medizinische Fakultät Carl Gustav Carus der TU, Dresden, Germany; ${ }^{9}$ Institut für Humangenetik, Universität Frankfurt, Germany; ${ }^{10}$ Institut für Humangenetik, Charité, Berlin, Germany; ${ }^{11}$ Institut für Humangenetik, Friedrich-Alexander-Universität, Erlangen-Nürnberg, Germany; ${ }^{12}$ Praxis für Humangenetik, Bremen, Germany; ${ }^{13}$ Klinik für Mund-, Kiefer- und Gesichtschirurgie, Universitätsklinikum Kiel, Germany; ${ }^{14}$ Institut für Humangenetik, Universität Göttingen, Germany

To define the range of phenotypic expression in Treacher Collins syndrome (TCS; Franceschetti-Klein syndrome), we performed mutation analysis in the TCOF1 gene in 46 patients with tentative diagnosis of TCS and evaluated the clinical data, including a scoring system. A total of 27 coding exons of TCOF1 and adjacent splice junctions were analysed by direct sequencing. In 36 patients with a clinically unequivocal diagnosis of TCS, we detected 28 pathogenic mutations, including 25 novel alterations. No mutation was identified in the remaining eight patients with unequivocal diagnosis of TCS and 10 further patients, in whom the referring diagnosis of TCS was clinically doubtful. There is no overt genotype-phenotype correlation except that conductive deafness is significantly less frequent in patients with mutations in the $3^{\prime}$ part of the open reading frame. Inter- and intrafamilial variation is wide. Some mutation carriers, parents of typically affected patients, are so mildly affected that the diagnosis might be overlooked clinically. This suggests that modifying factors are important for phenotypic expression. Based on these findings, minimal diagnostic criteria were defined: downward slanting palpebral fissures and hypoplasia of the zygomatic arch. The difficulties in genetic counselling, especially diagnosis of family members with a mild phenotype, are described.

European Journal of Human Genetics (2004) 12, 879-890. doi:10.1038/sj.ejhg.5201260

Published online 1 September 2004

*Correspondence: DR Lohmann, Institut für Humangenetik, Universitätsklinikum, 45122 Essen, Germany. Tel. + 49201 7234562; Fax + 49201 7235900; E-mail: dr.lohmann@uni-essen.de Authors 6-23 are listed alphabetically.

Received 23 March 2004; revised 26 May 2004; accepted 8 June 2004 


\section{Introduction}

Treacher Collins syndrome (TCS, MIM 154500) is an autosomal dominant mandibulofacial dysostosis. It is named after $\mathrm{E}$ Treacher Collins. ${ }^{1}$ However, there are two earlier reports by Thomson $(1846)^{2}$ and Berry (1889). ${ }^{3}$ The synonym Franceschetti-Klein syndrome is preferred in many German-speaking countries because of the excellent overview by Franceschetti and Klein (1949). ${ }^{4}$ TCS is diagnosed in 1/50000 live births. ${ }^{5,6}$ More than $60 \%$ of patients have no positive family history. ${ }^{7}$ TCS is characterized by bilateral downward slanting palpebral fissures, frequently accompanied by colobomas of the lower eyelid and a paucity of eyelashes medial to the defect, abnormalities of the external ears, atresia of external auditory canal and bilateral conductive hearing loss, hypoplasia of the zygomatic complex and mandible, and cleft palate. ${ }^{8,9}$ This complex pattern of malformation is thought to be caused by impaired development of structures derived from the first and second branchial arches between the 5th and 8th week of embryonic development. ${ }^{10}$ Patients with TCS were found to be heterozygous for mutations in the TCOF1 gene, which is located in $5 q 32-q 33.1 .^{11-18}$ This gene codes for a protein of at least 1411 amino acids, treacle, which is a nucleolar phosphoprotein that travels between nucleolus and cytoplasm. Treacle is composed of a 213 residue $\mathrm{N}$ terminus that is followed by 11 repeated units with potential phosphorylation sites and a C-terminus with multiple putative nuclear (NLS) and nucleolar localisation signals (NoLS). ${ }^{14,19}$ The pathogenetic role of TCOF1 mutations in TCS indicates that treacle is important for craniofacial development. Specifically, it was suggested that correct expression of treacle is essential for survival of cephalic neural crest cells. ${ }^{20}$

To date, more than 129 different mutations have been reported in patients with TCS. However, little is known about the range of phenotypic expression in carriers of TCOF1 gene mutations. To address this, we have investigated 36 patients with unequivocal diagnosis of TCS and 10 patients with mandibulofacial abnormalities compatible with a tentative diagnosis of TCS. We identified TCOF1 mutations in 28 of 36 (78\%) patients with the diagnosis of TCS. Downward slanting palpebral fissures and hypoplasia of the zygomatic complex were the only clinical findings present in each of these patients. Intriguingly, mutation analysis showed that some clinically unaffected parents are heterozygous mutation carriers. This shows that TCOF1 mutations can be associated with typical TCS and with phenotypes that are well within normal variation.

\section{Patients and methods Patients}

In response to a 'call for patients', 46 patients with a tentative diagnosis of TCS were referred to us. In total, 15 patients were examined at the Institut für Humangenetik, Universitätsklinikum Essen, and clinical data were obtained within the counselling session. Clinical examination of the remaining 31 patients was performed in outside hospitals and clinical data were reported using standardized questionnaires and photographs. We evaluated the clinical diagnosis of TCS of all patients referred to us. In all, 36/46 patients showed the characteristic facial 'gestalt' with typical clinical findings including downward slanting palpebral fissures, lower eyelid coloboma, hypoplasia of zygomatic complex and mandible, and microtia (Table 3). We developed a scoring system to quantify phenotypic expression in patients with clinically unequivocal diagnosis of TCS. The main clinical features (downward slanting palpebral fissures, lower eyelid coloboma, hypoplasia of zygomatic complex, hypoplasia of mandible, microtia) scored two points each, and accessory features (cleft palate, atresia of external ear canal, conductive deafness, tracheostoma, choanal stenosis/atresia, preauricular tags, delayed motor development, delayed speech development) scored one point each. The overall facial phenotype was classified as mild (scored with one point) or severe (scored with two points) based on photographs viewed by three of us (BA, GG-K, DW). The maximum sum of scores is 20 points if all relevant data are available. Patients with a score of $0-10 / 20$ are regarded as mildly affected and patients with scores of $11 / 20$ or more are regarded as severely affected. According to this scoring system, 9/35 patients are classified as mildly and $26 / 35$ as severely affected. In one patient, the scoring system was not applicable as important clinical data were not available. In a few patients, available data were incomplete and, therefore, maximum score in these patients is lower than 20 points (Table 3).

We tried to obtain clinical data, photographs, and blood samples from parents of patients. On the basis of clinical data, parents of six patients also had a TCS phenotype.

In $10 / 46$ patients, we could not agree with the referring diagnosis because the main clinical features (see above) were missing or because of the presence of additional clinical findings atypical of TCS, such as short stature, anophthalmia, syndactyly of fingers III/IV, and tracheoesophageal fistula. Discussed differential diagnosis in these patients were oculo-auriculo-vertebral spectrum (OAVS), ${ }^{21}$ CHARGE association, ${ }^{22}$ autosomal recessive 
Table 1 Sequences of oligonucleotidprimers for PCR

\begin{tabular}{|c|c|c|c|c|}
\hline Exon & Sense primer $\left(5^{\prime} \rightarrow 3^{\prime}\right)$ & Antisense primer $\left(5^{\prime} \leftarrow 3^{\prime}\right)$ & Product size $(b p)$ & Reference \\
\hline 1 & Aagtggggcgcgcgaggt & Cacacggcccacgaacgctt & 224 & 13 \\
\hline 2 & Ccaaaaagaccctcttctga & Tatcccagcttattccaagc & 251 & \\
\hline 3 & Agttgttctgtcacccagttg & Ctccccagggtcttttaggt & 299 & \\
\hline 4 & Gcatcacagagctcattcct & Gtggggcaattactcacaac & 343 & \\
\hline 5 & Agttcaccatgccataccag & Ttccagaaatgtcccagaac & 329 & \\
\hline 6 & Ctttgatgagcagctggttt & Accctaccacagttgctgaa & 318 & \\
\hline $6 \mathrm{~A}$ & Cagaaccttagggggaaacag & Tagtcctccctctccccaac & 400 & \\
\hline $7-13$ & Aaggcctctggactttatcc & Cagtcaagcccatcctca & 2603 & \\
\hline $14-16$ & Atctcaccttctccctcctt & Ccgttctgaggagtgagtgt & 912 & \\
\hline $16 \mathrm{~A}$ & Ccgaccacgtgcttatcc & Atggcgagattttccctatg & 246 & \\
\hline $17-18$ & Accctttgccttgtaaaaca & Catcagcacatgtgggtaagt & 2296 & \\
\hline $19-22$ & Gagtagtggggcctaagctg & Atcttcaggtccaatgcaag & 2399 & \\
\hline 23 & Agatctgtcccccaactctc & Tacatgggaggaatgagacc & 714 & 13 \\
\hline 24 & Gttgtgatggcttctggtg & Caggtctgggtgtgtaggag & 294 & \\
\hline 25 & Cagggcctcagctgtattta & Cctgaatgctcaggaatgat & 254 & \\
\hline
\end{tabular}

bilaterial microtia with conductive deafness, ${ }^{23}$ Feingold syndrome, ${ }^{24}$ and ROCA syndrome. ${ }^{25}$

In $9 / 10$ of these patients, only the index patient was affected. In one patient and his brother, we established the diagnosis Burn-McKeown syndrome. ${ }^{26,27}$

\section{Mutational analysis}

Genomic DNA was extracted from blood samples using Nucleon DNA extraction kits (Amersham Health, Ismaning, Germany). We used direct sequencing to identify mutations in the 27 exons and flanking splice sites of the TCOF1 gene. Four different PCR reactions were used to cover regions containing multiple adjacent exons (7-13, 14-16, 17-18, and 19-22) (Table 1). Internal primers were used for sequencing of PCR products spanning more than one exon (Table 2). Exons 1-6A, 16A and 23-25 were analyzed exon-by-exon. Primer sequences used in this study were adopted from the literature or chosen using the program 'primer 3' (http://www-genome.wi. mit.edu/cgi-bin/primer/primer3_www.cgi). Oligonucleotides were custom sythesized by Eurogentec (Köln, Germany).

Except for exons 1 and 4, PCR was performed in $50 \mu \mathrm{l}$ with $200 \mathrm{ng}$ genomic DNA, $5 \mu \mathrm{l} 10 \times$ PCR buffer (with $\left.1.5 \mathrm{mM} \mathrm{MgCl}_{2}\right), 8 \mu \mathrm{l}$ dNTP $(125 \mu \mathrm{M}), 15$ pmol of sense and antisense primer $(20 \mu \mathrm{M})$, and $1 \mathrm{U}$ AmpliTaq polymerase. We used a touchdown thermal cycling profile with initial denaturation at $95^{\circ} \mathrm{C}$ for $2 \mathrm{~min}$ followed by 14 cycles of $94^{\circ} \mathrm{C}$ for $20 \mathrm{~s}, 63^{\circ} \mathrm{C}$ for $1 \mathrm{~min}\left(-0.5^{\circ} \mathrm{C}\right.$ per cycle), $72^{\circ} \mathrm{C}$ for $1 \mathrm{~min}$, followed by 20 cycles $94^{\circ} \mathrm{C}$ for $20 \mathrm{~s}, 56^{\circ} \mathrm{C}$ for $1 \mathrm{~min}$, and, finally, $72^{\circ} \mathrm{C}$ for $1 \mathrm{~min}$ (Gene Amp 9700 Thermal Cycler, Perkin-Elmer). For PCR amplification of exon 4, we used half the concentration of primers and the start temperature of the touchdown profile was set to $65^{\circ} \mathrm{C}$. For amplification of exon 1, we used the Advantage-GC2PCR Kit (Clontech, Heidelberg, Germany) and the following thermal cycling profile: initial
Table 2 Sequences of oligonucleotidprimers for sequencing

\begin{tabular}{llll}
\hline Exon & Oligonucleotid sequence & Direction & Reference \\
\hline 1 & Gggcgcgagggaagtg & Sense \\
2 & Tatccagcttattccaagc & Antisense \\
3 & Agttgttctgtcacccagttg & Sense \\
4 & Caatagaattgttaggtgag & Sense \\
5 & Agttcaccatgccataccag & Sense \\
6 & Ctttgatgagcagctggttt & Sense \\
$6 \mathrm{~A}$ & Tagtcctcctctcccaac & Antisense \\
7 & Aaggcctctggactttatcc & Sense \\
8 & Gtgtcctgtgtctcctcac & Sense \\
9 & Tctttgaggcagggcacag & Antisense \\
$10 \mathrm{a}^{\mathrm{a}}$ & Actcctccctaatcttgtc & Sense \\
$10 \mathrm{~b}^{\mathrm{a}}$ & Gaaagagccttacaggaagg & Antisense \\
$11 \mathrm{a}^{\mathrm{a}}$ & Ctctcccgatcctgtgtatc & Sense \\
$11 \mathrm{~b}^{\mathrm{a}}$ & Atcaggccatgtagcagcct & Antisense \\
12 & Tctccagcctttcttggtg & Sense \\
13 & Ggatgggcctgctccttcta & Antisense \\
14 & Atctcaccttctcctcctt & Sense \\
15 & Ggagtgggacctgaaagaat & Sense \\
16 & Ccgttctgaggagtgagtgt & Antisense \\
$16 \mathrm{~A}$ & Atggcgagatttccctatg & Antisense \\
17 & Agcttctagccactggtctg & Antisense \\
18 & Ccagagcagccatttgag & Sense \\
$19-20$ & Tctgagatatggagctgagg & Antisense \\
21 & Ggacctgcagagagaccag & Sense \\
22 & Acccactctgcactgatagg & Sense \\
$23 \mathrm{a}^{\mathrm{a}}$ & Tcagtggtgaaggtcctgac & Sense \\
$23 \mathrm{~b}^{\mathrm{a}}$ & Caagagaggggttcccttc & Antisense \\
24 & Gttgtgatggcttctggtg & Sense \\
25 & Cagggcctcagctgtattta & Sense \\
\hline
\end{tabular}

${ }^{a}$ Exons were sequenced in both directions.

denaturation at $95^{\circ} \mathrm{C}$ for $2 \mathrm{~min}, 35$ cycles of $95^{\circ} \mathrm{C}$ for $15 \mathrm{~s}$, $69^{\circ} \mathrm{C}$ for $15 \mathrm{~s}$, followed by $72^{\circ} \mathrm{C}$ for $30 \mathrm{~s}$. For $\mathrm{PCR}$ amplification of exon $6 \mathrm{~A}$ and $16 \mathrm{~A}$, we used the following thermal cycling profile: initial denaturation at $96^{\circ} \mathrm{C}$ for $5 \mathrm{~min}, 35$ cycles of $96^{\circ} \mathrm{C}$ for $30 \mathrm{~s}, 72^{\circ} \mathrm{C}$ for $30 \mathrm{~s}$, followed by $72^{\circ} \mathrm{C}$ for $7 \mathrm{~min}$. 
a
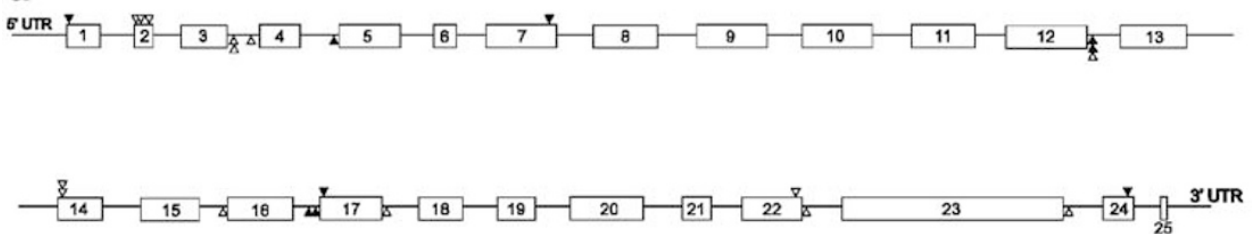

b
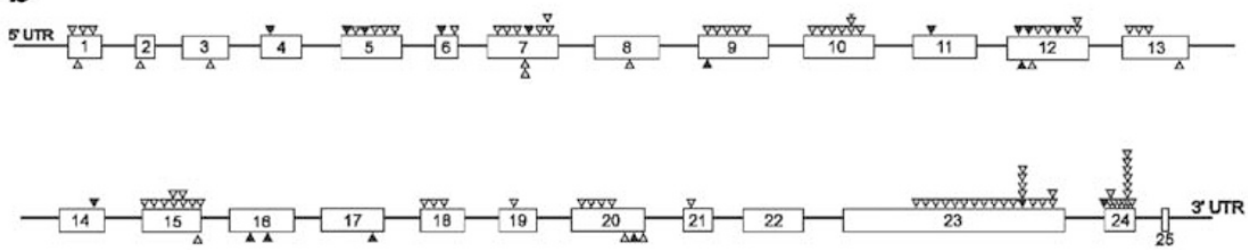

Figure 1 TCOF1 gene with newly identified and previously reported mutations. The exons are scaled according to their size, introns are not scaled. (a) TCOF1 gene with nonsense and frameshift mutations (arrowhead at the bottom: nonsense mutations; arrowhead at the top: frameshift mutations; black: newly identified mutations; gray: previously described mutations. ${ }^{11-17}$ (b) TCOF1 gene with splice and missense mutations (arrowhead at the bottom: splice mutations; arrowhead at the top: missense mutations; black: newly identified mutations; gray: previously described mutations. ${ }^{11-17}$

\section{Statistical analysis}

Statistical analysis was performed using the software package R (http://www.r-project. org). The relationship of individual polymorphisms with mild versus severe phenotype was tested using Fisher's exact test in a 2-by-2 contingency table. To analyze individual symptoms or the derived score with respect to mutation positions, a linear regression was performed.

\section{Results \\ TCOF1 gene mutations in patients with TCS}

We identified heterozygous mutations in 28/36 (78\%) patients with unequivocal diagnosis of TCS (Table 4). Only two of these mutations have been reported previously (c.728insC and c.3987insG). ${ }^{12,13}$ Two unrelated patients in our study showed the identical mutation (c.1911 +1G >A, patients M12737 and M20178). The local distribution of the mutations showed no clustering in specific regions of the TCOF1 gene (Figure 1). Most of the mutations are predicted to lead to premature termination codons because of frameshift length alterations (mutations in 14 patients) or nonsense substitutions (mutations in six patients). Mutations identified in five patients affected invariable bases at splice donor $(n=2)$ or acceptor $(n=3)$ sites. One of these, the splice mutation identified in patient M17995 (c.2629-3A $>\mathrm{G}$ ) does not change an invariable base. However, analysis of RNA from peripheral blood from this patient showed that this mutation activates a cryptic splice acceptor site in exon 17 and results in a transcript with frameshift deletion (data not shown). This mutation was not detected in 200 normal chromosomes.
Two patients were heterozygous for missense mutations (S266N, patient M19449; V877M, patient M18658). None of the two mutations alter amino acids that are conserved in known orthologs of TCOF $1 .^{28,29}$ Both mutations were not detected in 200 normal chromosomes. Sequence analysis in parents of these patients showed that the mutation V877M occurred de novo, and that the missense change S266N was inherited from the mother. It remains unclear if she has a TCS phenotype because sufficiently detailed clinical data were not available. One patient showed a single-base substitution that abolishes the initiation codon (ATG1ATA, patient M17639). In other genes, mutations of this kind have been found to severely disturb correct translation initiation and usually are associated with a phenotypic expression similar to that of null mutations (HBB, CFTR). ${ }^{30,31}$ We also identified no mutation in the recently described exons, ${ }^{19} 6 \mathrm{~A}$ and $16 \mathrm{~A}$, in these patients.

We identified no TCOF1 gene mutation in any of the 10 patients with tentative diagnosis of TCS.

Genotype-phenotype associations in index patients We had to exclude one patient (M18773) with a known mutation (c.3987insG) from the genotype-phenotype correlation because relevant clinical data were missing.

A TCOF1 mutation was identified in 19/26 (73\%) isolated cases and five of six (83\%) patients with a positive family history. Figure 2 shows pictures of the faces of these patients. There is a wide variation of the facial 'gestalt' between patients from different families. The phenotype scores, as defined above, varied accordingly. 


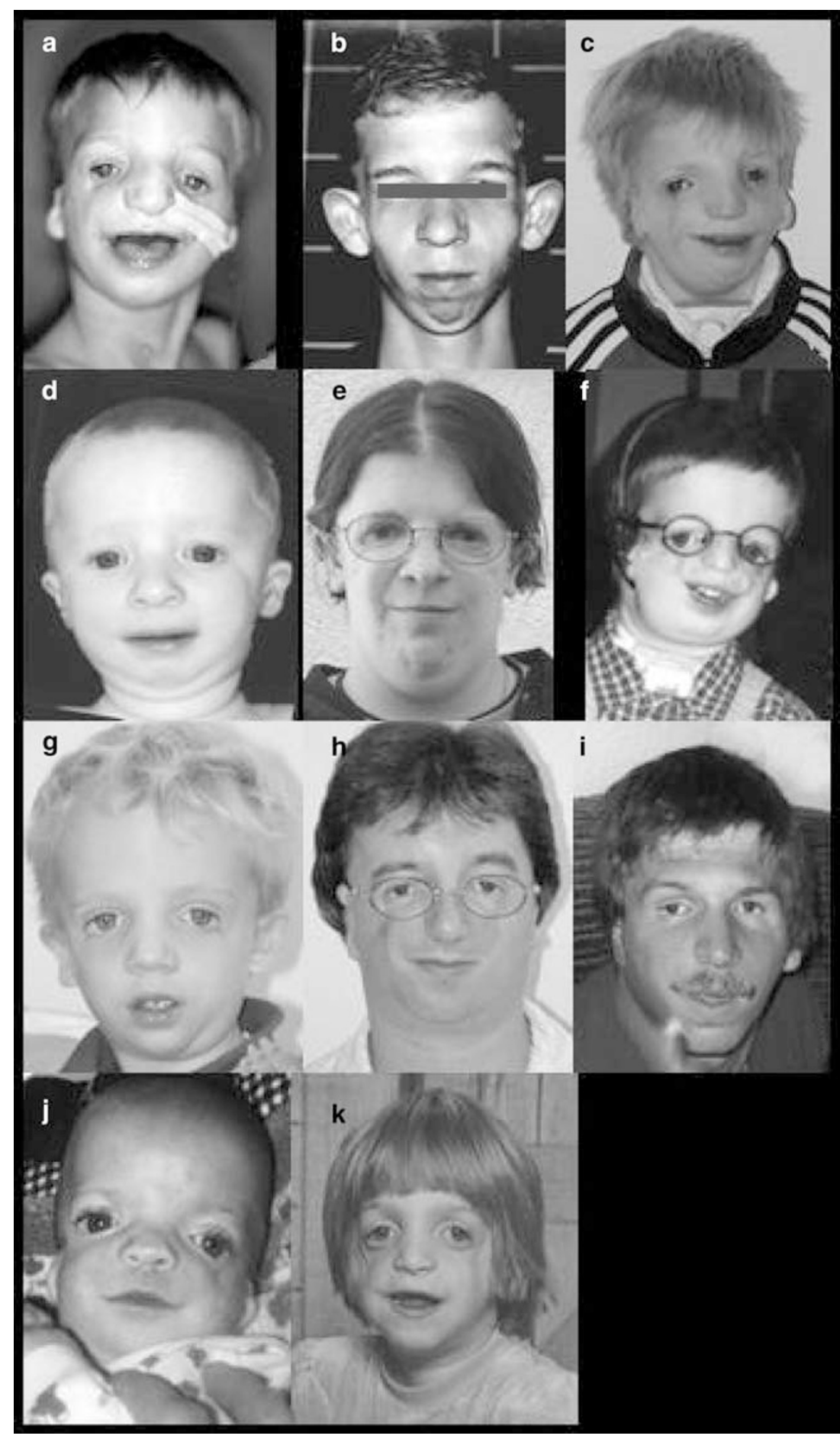

Figure 2 Patients with TCS and detected TCOF1 mutation, (a-k) are arranged according to the location of the mutation in the TCOF1 gene. (a) Patient M17639 with M1I, (b) patient M17807 with 121X, (c) patient M20194 with 209X, (d) patient M19731 with K367X, (e) patient M18982 with Q563X, (f) patient M18923 with 795X, (g) patient M17629 with Q818X, (h) patient M18013 with 854X, (i) patient M18774 with G848X, (j) patient M17995 with c.2629-3A>G, (k) patient M18293 with $1392 \mathrm{X}$ 


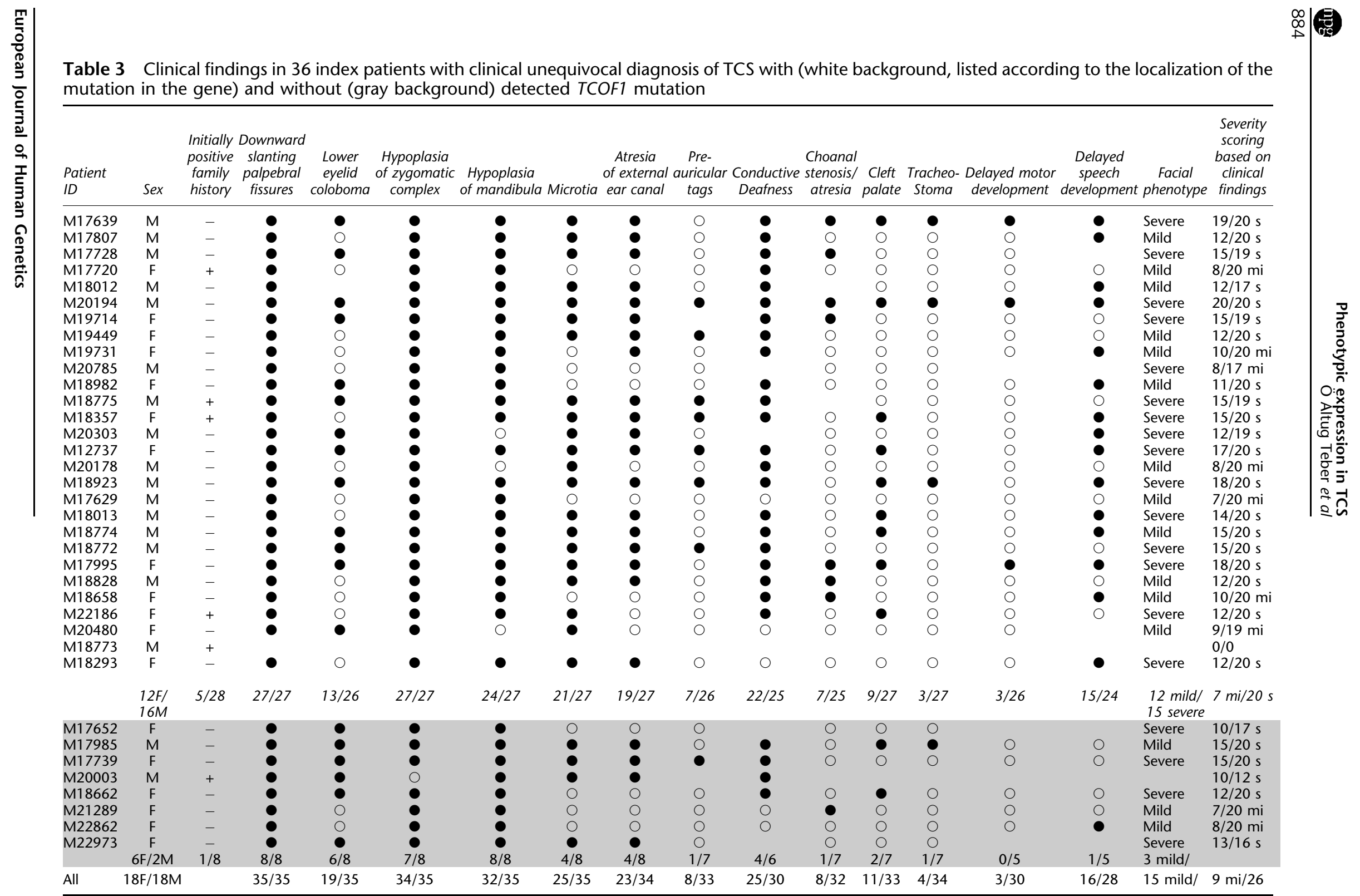

- present; $\bigcirc$ : absent; no symbol: clinical data unavailable; mi: mild phenotype (listed clinical findings present in less than $50 \%$ ); s: severe phenotype (listed clinical findings present in more than $50 \%)$. 


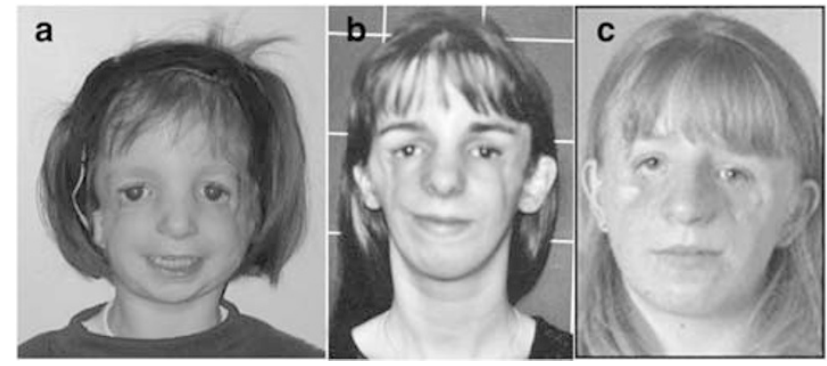

Figure 3 Three of the eight patients with a clinical unequivocal diagnosis of TCS without detected TCOF1 mutation. (a) patient M17739, (b) patient M18662, (c) patient M17652.

Rates of mutation detection are similar in patients with a mild 7/9 (78\%) and with a severe TCS phenotype 20/26 (77\%). Mutations predicted to result in premature termination codons are frequent both in mildly $(5 / 7,71 \%)$ and in severely affected patients $(15 / 20,75 \%)$. The proportion of splice site mutations is also similar in patients with mild $1 /$ 7 (14\%) and severe 4/20 (20\%) phenotypic expression. Interestingly, the percentage of missense mutations is different (1/20 (5\%) in severely affected and $1 / 7(14 \%)$ in mildly affected patients). The locations of the mutations identified in mildly and severely affected patients show no distinct pattern. When comparing the location of the mutation within the TCOF1 gene with the presence or absence of individual clinical findings as listed in Table 3, we observed that conductive deafness is significantly $(P=0.0099)$ less frequent in patients who have mutations in the $3^{\prime}$ part of the open reading frame. However, this needs to be confirmed by analysis of independent data sets.

The pattern of clinical findings in patients with unequivocal diagnosis of TCS but no TCOF1 gene mutation was not distinct from those with a known mutation (Table 3, Figure 3).

\section{Intrafamilial variation of phenotypic expression}

Blood DNA from both parents was available for mutation analysis in 14 of the 24 patients diagnosed with isolated TCS. Parents of 10 of these patients (71\%) did not show the mutation identified in their child and, therefore, the mutation occurred de novo.

Interestingly, four clinically unaffected parents (two mothers, two fathers) were found to be heterozygous. In addition, two affected relatives of patients with familial TCS (M18357 and M22186) were found to be heterozygous for the mutation identified in the respective index patient. In all, six families were available for analysis of intrafamilial variation.

Clinical data required for the analysis of intrafamilial phenotypic variation were available from five of these families (Figure 4). In one family (M17629), phenotypic expression was mild in all affected family members (Figure 4a). In another family (M18357), both mother and daughter were severely affected (Figure $4 \mathrm{~b}$ ). In the remaining three families, a parent was recognized as affected only after diagnosis in a severely affected child prompted clinical investigation of the parents (Figure 4c, d). In familial cases, there were three transmissions from carrier fathers and three transmissions from carrier mothers (Table 4). The parental origin of a mutation did not influence phenotype: two of three paternally inherited and three of three maternally inherited mutant alleles are associated with a severe phenotype.

\section{Polymorphic variants of the TCOF1 gene in patients with TCS}

In the course of mutation analysis, we identified 32 different SNPs in the TCOF1 gene (Table 5). A total of 21 of them have not been reported previously. These include four cSNPs (T168M, G256G, E410E, P881P, insK1382). The first three expressed variants are likely to be rare as each was identified only in one patient, the fourth one was also found in one control sample.

\section{Discussion}

Historically, diagnosis of malformation syndromes is based on the clinical identification of a specific pattern of phenotypic abnormalities. Often, the establishment of the diagnosis is guided by a characteristic facial phenotype. Recently, the genetic causes of several of these developmental disorders have been elucidated. With the possibility of a molecular diagnosis, the range of phenotypic expression of a given syndrome can be determined with less bias because patients with a nonclassical phenotype can be included in the spectrum if they show a pathological genotype that has also been observed in patients with regular phenotypic expression. It is to be expected that analysis of genotype-phenotype associations will help to define more specific criteria for clinical diagnosis. One goal of the present study was to define the range of phenotypic expression in TCS. The proportion of patients in which we identified a mutation compares well to that of previous reports. ${ }^{11-16}$ However, the spectrum of mutations in our patients is somewhat different. For example, none of our patients carries the deletion nt4135del(GAAAA) in exon 24 of the TCOF1 gene although, in previous reports, this mutation was identified in $20 \%$ of TCS patients. ${ }^{17}$ Moreover, we identified significantly more single-base substitutions: 14 of $28(50 \%)$ mutations in this study compared to 24 of $121(19.8 \%)$ in previous reports (Fisher's test: $P=0.02782) .{ }^{11-13,15-17}$ Possibly, this difference is a consequence of the methods that were used for mutation detection. We have used direct sequencing which, com- 
a

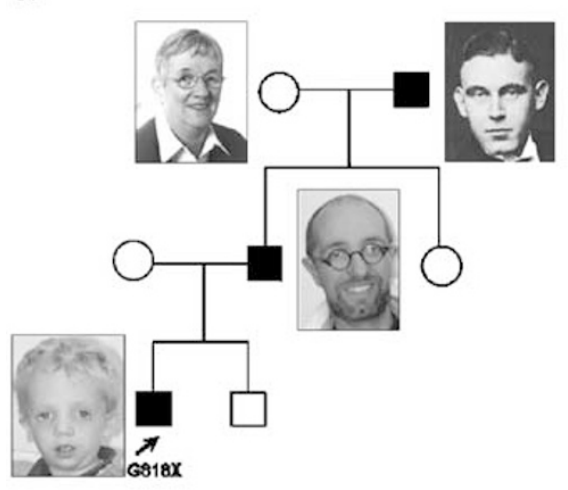

C

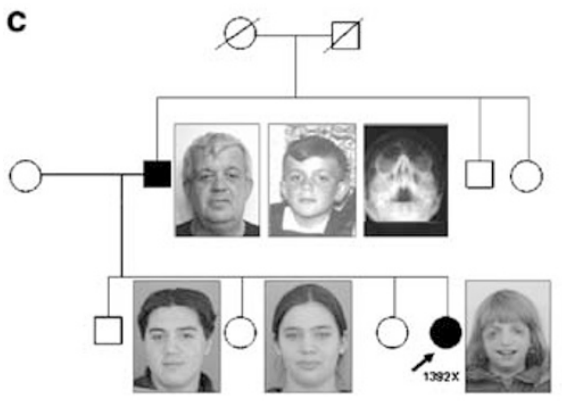

b
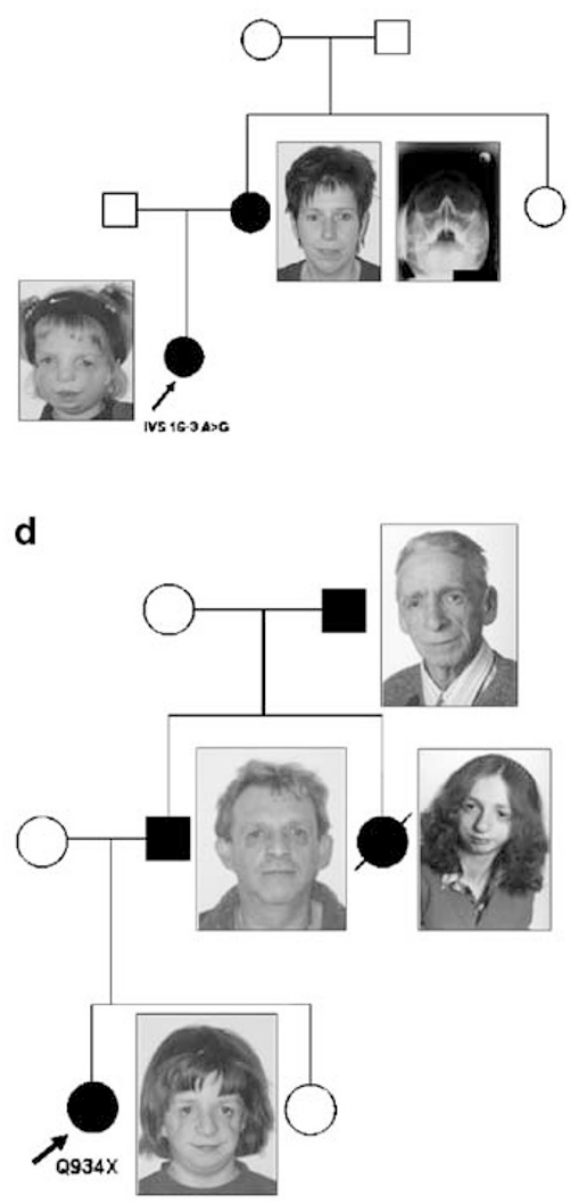

Figure 4 Intrafamilial variation. (a) Pedigree of family M17629. The proband shows the characteristic facial phenotype with downward slanting palpebral fissures, hypoplastic zygomatic complex, slightly dysplastic ears, conductive hearing loss, and hypoplasia of mandibula. A stop mutation (G818X) was identified to be the causative mutation. The proband's father was much milder affected and his beard and his glasses mask the phenotype: conductive hearing loss was lacking and his ears were surgically corrected. The paternal grandmother has a facial phenotype similar to his son and a positive family history for dysplastic ears. Surprisingly, the paternal grandfather, who has no facial characteristics for TCS, carries the mutation. He might be an example of nonpenetrance, although he denied personal investigation and radiographs. (b) Pedigree of family M17995. The proband is severely affected with hypoplasia of the zygomatic complex, bilateral microtia with atresia of the external auditory canal, cleft palate, bilateral choanal atresia. A splice mutation (c.2629-3A>G) seems to be causative in this patient. The mother has a hypoplasia of the mandible and clinically suspicion of hypoplasia of right zygomatic complex, although she has a normal slant of palpebral fissures. Radiographic examination (Waters's projection) clearly shows the hypoplasia of the zygomatic complex. We consider that the mother is mildly affected. (c) Family M18293. The proposita is severely affected with bilateral microtia, hypoplastic zygomatic complex, antimongoloid slant of palpebral fissures. We received some photographs of the father and suggested that he was not affected. After molecular investigation and knowing him as a mutation carrier, we were able to personally investigate him. The only abnormal facial findings were his slightly downward slanting palpebral fissures. It is much easier to recognize the mild facial phenotype in childhood. Waters's projection showed bilateral hypoplasia of zygomatic complex with unilateral left-sided aplastic zygomatic arch. The brother and the elder sister of the proposita do not carry the mutation. (d) Family M22186. The proposita is severely affected. Her father only shows mild hypoplasia of the zygomatic complex. He himself believed that he was unaffected, diagnosis was established after birth of his affected daughter. His sister was severely affected (not molecularly proved) and died at the age of 20 years due to cardiac insufficiency. The paternal grandfather has a mild hearing loss and downward slanting palpebral fissures. He is most likely mildly affected, but DNA was not available. 
Table 4 Summary of TCOF1 gene mutations detected in this study

\begin{tabular}{|c|c|c|c|c|c|c|c|}
\hline & Patient ID & Mutation & Type & Location & Effect & Inherited & Recurrent \\
\hline 1 & M17639 & c. $3 \mathrm{G}>\mathrm{A}$ & d & Exon 1 & & - & - \\
\hline 2 & M17807 & c.343delG & $a$ & Exon 4 & $121 X$ & Not tested & - \\
\hline 3 & M17728 & c. $379-2 A>G$ & b & Intron 4 & Splice & - & - \\
\hline 4 & M17720 & c.390delGA & $a$ & Exon 5 & $173 x$ & Not tested & - \\
\hline 5 & M18012 & c.408delG & $a$ & Exon 5 & $214 X$ & Not tested & - \\
\hline 6 & M20194 & c.574del(16bp) & $a$ & Exon 6 & $209 X$ & - & - \\
\hline 7 & M19714 & c.728insC & $a$ & Exon 7 & $271 X$ & Not tested & +12 \\
\hline 8 & M19449 & c.797G > A & $\mathrm{C}$ & Exon 7 & S266N & Maternally & - \\
\hline 9 & M19731 & c. $1099 \mathrm{~A}>\mathrm{T}$ & a & Exon 9 & K367X & - & - \\
\hline 10 & M20785 & c. $[1552$ delG; $1565 \mathrm{~T}>\mathrm{C}]$ & a & Exon 11 & $518 X$ & - & - \\
\hline 11 & M18982 & c. $1687 \mathrm{C}>\mathrm{T}$ & a & Exon 12 & Q563X & Not tested & - \\
\hline 12 & M18775 & c.1742insC & a & Exon 12 & $620 x$ & Not tested & - \\
\hline 13 & M18357 & c.1768insC & a & Exon 12 & $620 x$ & Maternally & - \\
\hline 14 & M20303 & c.1868delATAG & a & Exon 12 & $632 X$ & Not tested & - \\
\hline 15 & M12737 & c. $1911+1 G>A$ & b & Intron 12 & Splice & - & - \\
\hline 16 & M20178 & c. $1911+1 \mathrm{G}>\mathrm{A}$ & b & Intron 12 & Splice & Not tested & - \\
\hline 17 & M18923 & c.[2205insTT; 2206delG] & a & Exon 14 & $795 X$ & - & - \\
\hline 18 & M17629 & c. $2452 \mathrm{C}>\mathrm{T}$ & a & Exon 16 & Q818X & Paternally & - \\
\hline 19 & M18013 & c.2526insA & a & Exon16 & $854 X$ & Not tested & - \\
\hline 20 & M18774 & c. $2542 C>T$ & a & Exon 16 & G848X & Not tested & - \\
\hline 21 & M18772 & c. $2545 \mathrm{delG}$ & a & Exon 16 & $872 X$ & Not tested & - \\
\hline 22 & M17995 & c. $2629-3 A>G$ & b & Intron 16 & Splice & Maternally & - \\
\hline 23 & M18828 & c. $2629-1 G>A$ & b & Intron 16 & Splice & - & - \\
\hline 24 & M18658 & c. $2629 \mathrm{G}>\mathrm{A}$ & C & Exon 17 & V877M & - & - \\
\hline 25 & M22186 & c. $2800 \mathrm{C}>\mathrm{T}$ & $a$ & Exon 17 & Q934X & Paternally & - \\
\hline 26 & M20480 & c. $3169 \mathrm{C}>\mathrm{T}$ & $a$ & Exon 20 & Q1057X & - & - \\
\hline 27 & M18773 & c.3987insG & $a$ & Exon 23 & $1352 X$ & Not tested & $+13-16$ \\
\hline 28 & M18293 & c.4122delCA & $a$ & Exon 24 & $1392 X$ & Paternally & - \\
\hline
\end{tabular}

a, mutation resulting in premature termination; b, splice site mutation; $c$, missense mutation, $d$, mutation in the translation initiating methionine.

pared to SSCP, has a higher sensitivity for the detection of point mutations.

We identified 25 novel mutations including a base substitution in the start codon (M1I). It is known from other genes that mutational change at the initiating codon can result in loss of normal gene function (HBB, CFTR). We identified this mutation in a patient with a severe TCS phenotype. This association is in line with the hypothesis of Splendore et al, ${ }^{16}$ who suggested that the N-terminal part of treacle is important for proper function and thus less tolerant of changes. We also identified two novel missense mutations, a V877M change that was the consequence of a de novo mutation and a $\mathrm{S} 266 \mathrm{~N}$ substitution, which was also detected in the patient's mother. Unfortunately, the mother did not consent to provide the clinical data needed to evaluate if she has TCS with mild expression. The missense change S266N is located in a conserved motif of exon 7, which is also found in nine other exons of the TCOF1 gene. This motif is thought to contain phosphorylation sites ${ }^{14}$ and therefore the substitution of a serine to an asparagine results in loss of a potential phosphorylation site. However, we must note that we cannot exclude the possibility that the $\mathrm{S} 266 \mathrm{~N}$ substitution is not the causative mutation but a rare neutral variant.

In seven of 36 patients with clinically unequivocal diagnosis of TCS, we could not detect any potentially disease-related TCOF1 gene alteration although we sequenced all exons, including the recently described exons $6 \mathrm{~A}$ and $16 \mathrm{~A}$, and intron regions adjacent to exons. We may have missed large deletions or point mutations in introns and regulatory regions that were not covered by sequence analysis. Mutations of this kind constitute a part of the mutation spectra of many genes but have not been identified in TCOF1 so far. However, it is reasonable to assume that this kind of alterations can occur in TCOF1, and thus may account for TCS in those of our patients without detectable point mutation.

We found that phenotypic expression in patients with molecularly confirmed diagnosis of TCS is variable. The most consistent findings in index patients are hypoplasia of the mandible $(24 / 27,89 \%)$, conductive deafness $(22 / 25$, $88 \%)$, any degree of microtia $(21 / 27,78 \%)$, and atresia of the external ear canal (19/27, 70\%). Interestingly, most family members with TCOF1 mutations have no ear anomalies, even if microtia in index cases is severe. This is in line with Splendore et $a l^{15}$ who reported that the frequency of external ear abnormalities in index patients in familial cases is nine times that of their affected relatives. Thus, microtia is not helpful for detecting mildly affected patients.

Less frequent findings are lower eyelid coloboma (13/26, $50 \%)$, cleft palate $(9 / 27,33 \%)$, choanal stenosis/atresia (7/ 
Table 5 Summary of SNPs in the TCOF1 gene identified in this study

\begin{tabular}{|c|c|c|c|}
\hline SNP & Location & Nucleotid change & Amino-acid change \\
\hline 1 & Exon 5 & c. $503 \mathrm{C}>\mathrm{T}^{\mathrm{b}}$ & T168M \\
\hline 2 & Intron 5 & c. $565+18 \mathrm{G}>\mathrm{C}^{\mathrm{b}}$ & \\
\hline 3 & Intron 6 & c. $639+32 C>T^{15}$ & \\
\hline 4 & Exon 7 & c. $768 \mathrm{G}>\mathrm{A}^{\mathrm{b}}$ & G256G \\
\hline 5 & Intron 7 & c. $852+39 \mathrm{~A}>\mathrm{G}^{\mathrm{b}}$ & \\
\hline 6 & Intron 8 & c. $1047+60 \mathrm{G}>\mathrm{C}^{\mathrm{b}}$ & \\
\hline 7 & Exon 9 & c. $1230 \mathrm{~A}>\mathrm{G}^{\mathrm{b}}$ & $\mathrm{E} 410 \mathrm{E}$ \\
\hline 8 & Exon 10 & c. $1347 \mathrm{~T}>\mathrm{C}^{14,15}$ & P449P \\
\hline 9 & Intron 10 & c. $1473+68 C>T^{b}$ & \\
\hline 10 & Exon 11 & c. $1530 \mathrm{G}>\mathrm{T}^{15}$ & G510G \\
\hline 11 & Exon 11 & c. $1552 \mathrm{G}>\mathrm{A}^{14}$ & V518I \\
\hline 12 & Exon 11 & c. $1611 G>A^{14,15}$ & S537S \\
\hline 13 & Exon 12 & c. $1762 C>G^{36}$ & P588A \\
\hline 14 & Intron 12 & c. $1911+36 \mathrm{del}^{\mathrm{b}}$ & \\
\hline 15 & Intron 14 & c. $2248-42$ ins $G^{b}$ & \\
\hline 16 & Intron 14 & c. $2247+27 \mathrm{G}>\mathrm{A}^{\mathrm{b}}$ & \\
\hline 17 & Intron 15 & c. $2428-20$ ins CTCTC ${ }^{14}$ & \\
\hline 18 & Intron 15 & c. $2428-36 \mathrm{~A}>\mathrm{C}^{\mathrm{b}}$ & \\
\hline 19 & Exon 16 & c. $2429 \mathrm{C}>\mathrm{T}^{13-15}$ & A810V \\
\hline 20 & Intron $16 \mathrm{~A}$ & exon $16 A+4 C>T^{a}$ & \\
\hline 21 & Intron 16 & c. $2628+26 \mathrm{~A}>\mathrm{G}^{\mathrm{b}}$ & \\
\hline 22 & Exon 17 & c. $2643 \mathrm{C}>\mathrm{T}^{\mathrm{b}}$ & P881P \\
\hline 23 & Intron 19 & c. $3066+66 \mathrm{C}>\mathrm{T}^{15}$ & \\
\hline 24 & Intron 19 & c. $3067-72 A>C^{b}$ & \\
\hline 25 & Intron 20 & c. $3287-34 G>A^{b}$ & \\
\hline 26 & Exon 21 & c. $3296 \mathrm{C}>\mathrm{G}^{15}$ & P1099R \\
\hline 27 & Intron 21 & c. $3370-3 C>T^{b}$ & \\
\hline 28 & Exon 23 & c. $3938 \mathrm{C}>\mathrm{T}^{13,15}$ & A1313V \\
\hline 29 & Exon 23 & c. $4061 \mathrm{G}>\mathrm{C}^{15}$ & G1354A \\
\hline 30 & Intron 23 & c. $4112-17 \mathrm{~T}>\mathrm{A}^{\mathrm{b}}$ & \\
\hline 31 & Intron 24 & c. $4209+42 C>A^{b}$ & \\
\hline 32 & Exon 24 & c. 4148 insGAA & insK1382 \\
\hline
\end{tabular}

${ }^{a}$ Four nucleotides $3^{\prime}$ of the last coding nucleotide of exon $16 \mathrm{~A}$ (this nomenclature was chosen to leave the old nomenclature for the other polymorphisms).

${ }^{b}$ Newly identified SNP.

$25,28 \%)$, preauricular tags $(7 / 26,27 \%)$, tracheostoma due to upper airway obstruction $(3 / 27,11 \%)$ and delayed motor development $(3 / 26,12 \%)$. As these are not consistent findings in patients with a molecularly confirmed diagnosis of TCS, they are less helpful for establishing a clinical diagnosis. Interestingly, we observed that three of the molecularly confirmed patients with a high score (17/ $20,18 / 20,19 / 20)$, and therefore severe phenotypic expression of TCS, presented with a psychomotor delay. Speech delay is probably caused by hearing loss, which is a frequent finding in TCS. Delay in motor development is uncommon in TCS and, to the best of our knowledge has not been described previously. We hypothesize that delay of motor development may be not uncommon in TCS patients who are severely affected.

In 10 of the patients sent to us, we found that diagnosis was debatable because of the lack of the typical facial gestalt and absence of the main clinical findings. In three of them, a differential diagnosis was established. We identified no mutation in any of these 10 patients. From this, we conclude that the diagnosis TCS in sporadic cases is unlikely if the minimal diagnostic criteria are missing, or if untypical clinical findings, for example, short stature, hemivertebrae, or microcephaly, are present. Moreover, TCS is less likely in patients who are asymmetrically affected. This is in agreement with the findings of Splendore et $a l^{32}$ who performed TCOF1 gene mutation analysis in 22 patients with first and second branchial archrelated disorders distinct from typical TCS.

We have devised a scoring system to parameterize the severity of phenotypic expression. Index patients who are heterozygous carriers of a pathogenic TCOF1 mutation show a wide spectrum of phenotypic expression with scores ranging from $7 / 20$ (partial phenotype) to $20 / 20$ (complete phenotype). We observed no association between expression of the TCS phenotype and the location of the mutation within the TCOF1 gene or its biological consequence (missense vs premature termination), thus confirming previous findings. ${ }^{13}$ Moreover, analysis of phenotypic expression of mutations in families shows that the identical mutant allele can be present in patients with an unequivocal phenotype as well as in relatives who show only few signs characteristic of TCS. For example, in the present study, two parents were found to be carriers of TCOF1 mutations, who, previous to genetic analysis, were suspected to be unaffected (family M17995 and M18293). They had a score of $4 / 20$. These findings show that variable expressivity in carriers of TCOF1 mutations is not a simple consequence of mutational heterogeneity. In addition, the sequence data of the parents were evaluated with regard to mosaicism, but no indication for mosaicism was found. ${ }^{33}$ In general, phenotypic expression of a monogenic disorder may be modified by the combined effect of genetic, environmental, and stochastic factors.

The phenotypic consequences of mutational change in TCOF1 could be modified by non-neutral polymorphic variation in genes that are active along the same developmental pathway. For example, treacle interacts with hNop56p, a component of the box C/D small nucleolar ribonucleoprotein complexes that direct $2^{\prime}$-O-methylation of pre-RNA during its maturation. ${ }^{34}$ It might be interesting to investigate whether polymorphic variation of hNop56p is associated with expression of the TCS phenotype. However, it is reasonable to assume that treacle is embedded in a network of interactions with other proteins, and, therefore, hNop56p is possibly one of the many candidate modifier loci.

In some autosomal dominant disorders, phenotypic expression is modified by the sex of the parent that has transmitted the mutant allele. In our series of patients, the parental origin of the mutant allele is known in six mutation carriers. We observed that two of three paternally inherited and three of three maternally inherited mutant alleles were associated with a severe phenotype and, therefore, our data do not support parent-of-origin effects. 
Finally, another kind of genetic influence that possibly could account for the wide variation of phenotypic expression is modification in and between the other allele. TCS is thought to be due to haploinsufficiency caused by heterozygous TCOF1 mutations. This suggests that genetic modification could act through variation in the function of the other allele. It is reasonable to assume that there is variation of normal TCOF1 function because the TCOF1 gene is rich in polymorphic variation including several expressed SNPs that change the amino-acid sequence (1315 and this study). In 14 completely sequenced TCS patients, we found no significant correlation (Fisher's test) between the presence or absence of polymorphic variants and phenotypic expression.

Despite the wide phenotypic variation of TCS, some clinical findings are consistently present in all patients, namely downward slanting palpebral fissures and hypoplasia of the zygomatic complex. In most patients, the abnormal slanting of the palpebral fissures can be recognized on photographs. However, childhood photographs may be helpful, because this feature may be no longer so distinct in adults. If suitable photographs are not available, clinical examination is necessary to identify this anomaly (Figure $4 \mathrm{~b}, \mathrm{c}$ ). The presence of a hypoplastic zygomatic complex may be missed by clinical evaluation. Radiographs, especially Waters's projection, are very helpful for identifying this feature (Figure $4 \mathrm{~b}, \mathrm{c}$ ). Although present in all of our patients, downward slanting palpebral fissures and hypoplasia of the zygomatic complex are nonspecific features as they are not uncommon in healthy individuals. Notwithstanding low diagnostic specificity, these features may serve as the minimal diagnostic criteria. It is noteworthy at this point that in the premolecular age, Franceschetti and Zwahlen ${ }^{35}$ stressed that the barest minimal, single, clinical manifestation can be the presence of merely a slight antimongoloid obliquity of the palpebral fissures. These minimal diagnostic criteria are also backed by our family analyses. We identified several mutation carrying relatives who only presented with minimal diagnostic criteria. These mutation carriers were ascertained only because molecular testing was prompted by the presence of a relative with more severe phenotypic expression. Possibly, the prevalence of carriers of TCOF1 mutations in the population is higher than estimated now because carriers that only show downward slanting palpebral fissures and a hypoplastic zygomatic complex are not included. This causes an ascertainment bias that may also explain why in three of the families analyzed here, the severity of phenotypic expression seems to increase from generation to generation.

\section{Acknowledgements}

We thank the patients and their families for their cooperation and Bernhard Horsthemke for continuous support. In addition, we thank
Wolfgang Engel for sending DNA and clinical data. This study was supported by grants for OAT (IFORES 107110-0) by the Medical Faculty, University of Essen, and from the DAAD (A0119701) and by grants from the Deutsche Forschungsgemeinschaft (WI 1440/6-1).

\section{References}

1 Treacher Collins E: Case with symmetrical congenital notches in the outer part of each lower lid and defective development of the malar bones. Trans Ophthalmol Sec UK 1900; 20: 90.

2 Thomson A: Notice of several cases of malformation of the external ear, together with experiments on the state of hearing in such persons. Month J Med Sci 1846; 7: 420.

3 Berry GA: Note on a congenital defect (?coloboma) of the lower lid. $R$ Lond Ophthalmol Hosp Rep 1889; 12: 255-257.

4 Franceschetti A, Klein D: The mandibulo-facial dysostosis. A new hereditary syndrome. Acta Ophthalmol (Copenh) 1949; 27: 143-229.

5 Rovin S, Dachi SF, Borenstein DB, Cotter WE: Mandibulofacial dysostosis, a familial study of five generations. J Pediatr 1964; 65: 215-221.

6 Fazen LE, Elmore J, Nadler HL: Mandibulo-facial dysostosis (Treacher-Collins syndrome). Am J Dis Child 1967; 113: 405-410.

7 Connor JM, Ferguson-Smith MA: Essential medical genetics. Oxford: Blackwell Scientific, 1988.

8 Marres HAM, Cremers WRJ, Dixon MJ, Huygen PLM, Joosten FBM: The Treacher Collins syndrome. A clinical, radiological, and genetic linkage study on two pedigrees. Arch Otolaryngol Head Neck Surg 1995; 121: 509-514.

9 Dixon M: Treacher Collins syndrome. J Med Genet 1995; 32 : 806-808.

10 Poswillo D: The pathogenesis of the Treacher Collins syndrome (mandibulofacial dysostosis). Br J Oral Surg 1975; 13: 1-26.

11 Gladwin AJ, Dixon J, Loftus SK et al: Treacher Collins syndrome may result from insertions, deletions or splicing mutations, which introduce a termination codon into the gene. Hum Mol Genet 1996; 5: 1533-1538.

12 Treacher Collins collaborative group: Positional cloning of a gene involved in the pathogenesis of Treacher Collins syndrome. Nat Genet 1996; 12: 130-136.

13 Edwards SJ, Gladwin AJ, Dixon MJ: The mutational spectrum in Treacher Collins syndrome reveals a predominance of mutations that create a premature termination codon. Am J Hum Genet 1997; 60: 515-524.

14 Wise CA, Chiang LC, Paznekas WA et al: TCOF1 gene encodes a putative nucleolar phosphoprotein that exhibits mutations in Treacher Collins syndrome throughout its coding region. Proc Natl Acad Sci 1997; 94: 3110-3115.

15 Splendore A, Silva EO, Alonso LG et al: High mutation detection rate in TCOF1 among Treacher Collins syndrome patients reveals clustering of mutations and 16 non-pathogenic changes. Hum Mut 2000; 16: 315-322.

16 Splendore A, Jabs EW, Passos-Bueno MR: Screening of TCOF1 in patients from different populations: confirmation of mutational hot spots and identification of a new missense mutation that suggests an important functional domain in the protein treacle. J Med Genet 2002; 39: 493-495.

17 Ellis PE, Dawson M, Dixon MJ: Mutation testing in Treacher Collins syndrome. J Orthodont 2002; 29: 293-297.

18 Marszalek B, Wisniewski SA, Wojcicki P, Kobus K, Trzeciak WH: Novel mutation in the 5 ' splice site of exon 4 of the TCOF 1 gene in the patient with Treacher Collins syndrome. Am J Med Genet 2003; 123A: 169-171.

19 So RB, Gonzalez B, Henning D, Dixon J, Dixon MJ, Valdez BC: Another face of the Treacher Collins syndrome (TCOF1) gene: identification of additional exons. Gene 2004; 328: 49-57.

20 Dixon J, Brakebusch C, Fässler R, Dixon MJ: Increased levels of apoptosis in the prefusion neural folds underlie the craniofacial 
disorder, Treacher Collins syndrome. Hum Mol Genet 2000; 9: $1473-1480$.

21 Rollnick BR, Kaye CI, Nagatoshi K, Hauck W, Martin AO: Oculoauriculovertebral dysplasia and variants: phenotypic characteristics of 294 patients. Am J Med Genet 1987; 26: 361-375.

22 Hall BD: Choanal atresia and associated multiple anomalies. J Pediatr 1979; 95: 395-398.

23 Mengel MC, Königsmark BW, Berlin CI, McKusick VA: Conductive hearing loss and malformed low-set ears, as a possible recessive syndrome. J Med Genet 1969; 6: 14-21.

24 Courtens W, Levi S, Verbelen F, Verloes A, Vamos E: Feingold syndrome: report of a new family and review. Am J Med Genet 1997; 73: 55-60.

25 Zampino G, Balducci F, Mariotti P, Dickmann A, Mastroiacovo P: Growth and developmental retardation, ocular ptosis, cardiac defect, and anal atresia: confirmation of the ROCA-Wiedemann syndrome. Am J Med Genet 2000; 90: 358-360.

26 Wieczorek D, Altug Teber Ö, Lohmann D, Gillessen-Kaesbach G: Two brothers with Burn-McKeown syndrome. Clin Dysmorphol 2003; 12: 171-174.

27 Burn J, McKeown C, Wagget J, Bray R, Goodship J: New dysmorphic syndrome with choanal atresia in siblings. Clin Dysmorphol 1992; 1: 137-144.

28 Haworth KE, Islam I, Breen $\mathrm{M}$ et al: Canine TCOF1; cloning, chromosome assignment and genetic analysis in dogs with different head types. Mamm Genome 2001; 12: 622-629.

29 Dixon J, Hovanes K, Shiang R, Dixon MJ: Sequence analysis, identification of evolutionary conserved motifs and expression analysis of murine tcof 1 provide further evidence for a potential function for the gene and its human homologue, TCOF1. Hum Mol Genet 1997; 6: 727-737.

30 Saba L, Meloni A, Sardu R, Travi M, Primignani P, Rosatelli MC: A novel beta-thalassemia mutation $(\mathrm{G} \rightarrow \mathrm{A})$ at the initiation codon of the beta-globin gene. Hum Mutat 1992; 1: 420-422.

31 Cheadle JP, Belloni F, Ferrari M, Millar-Jones L, Meredith AL: A novel mutation (M1V) in the translation initiation codon of the cystic fibrosis transmembrane conductance regulator gene, in three CF chromosomes of Italian origin. Hum Mol Genet 1994; 3: $1431-1432$.

32 Splendore A, Passos-Bueno MR, Jabs EW, van Maldergem L, Wulfsberg EA: TCOF1 mutations excluded from a role in other first and second branchial arch-related disorders. Am J Med Genet 2002; 111: 324-327.

33 Hall JG: Review and hypothesis: somatic mosaicism: observations related to clinical genetics. Am J Hum Genet 1998; 43: 355-363.

34 Hayano T, Yanagida M, Yamauchi Y, Shinkawa T, Isobe T, Takahashi N: Proteonomic analysis of human Nop56-associated pre-ribosomal ribonucleoprotein complexes : possible link between Nop56p and the nucleolar protein treacle responsible for Treacher Collins syndrome. I Biol Chem 2003; 278: 34309-34319.

35 Franceschetti A, Zwahlen P: Un syndrome nouveau: la dysostose mandibulofaciale. Bull Schweiz Akad Med Wiss 1944; I: 60-66.

36 Haga H, Yamada R, Ohnishi Y, Nakamura Y, Tanaka T: Gene-based SNP discovery as part of the Japanese millenium genome project: identification of 190562 genetic variations in the human genome. J Hum Genet 2002; 47: 605-610. 